\title{
ESTIMULAÇÃO CEREBRAL PROFUNDA NO NÚCLEO SUBTALÂMICO PARA DOENÇA DE PARKINSON
}

\author{
José Augusto Nasser ${ }^{1,2}$, Asdrubal Falavigna' ${ }^{2}$, Armando Alaminos ${ }^{1}$, \\ Antônio Bonatelli3, Fernando Ferraz ${ }^{3}$
}

\begin{abstract}
RESUMO - Objetivo: Apresentamos os resultados preliminares da estimulação cerebral profunda crônica no núcleo subtalâmico para os sintomas motores da doença de Parkinson. Método: 8 pacientes com doença de Parkinson há mais de 5 anos, apresentando tremor incapacitante, oligocinesia e rigidez acentuada com predomínio unilateral, 3 à direita e um à esquerda, refratários à medicação adequada, foram submetidos ao implante bilateral de eletrodo cerebral profundo permanente subtalâmico, Itrell 2. Estes pacientes foram avaliados segundo as escalas: UPDRS, Schwab e England. Resultados: Os resultados preliminares (6 meses), mostraram melhora significativa dos sinais e sintomas avaliados pelas escalas acima. Não houve complicações com os procedimentos. Conclusão: Os resultados preliminares indicam que a estimulação subtalâmica é uma boa opção terapêutica, estando de acordo com os resultados da literatura.
\end{abstract}

PALAVRAS-CHAVE: doença de Parkinson, estimulação cerebral profunda, núcleo subtalâmico, estereotaxia.

\begin{abstract}
Deep brain stimulation of subthalamic nucleous in Parkinson's disease
ABSTRACT - We present the preliminary results in patients well selected to be implanted by deep brain stimulation (DBS) for Parkinsons's disease (PD). Methods: 8 PD patients with disabled tremor, akinetic/bradikinetic and rigidity, in spite of best therapeutic assay with poor response were referred to surgery. It was implanted DBS Itrell 2. Theses patients were evaluated by the following scales: UPDRS, Schwab and England. Results: The preliminary results in 6 months showed significant improvement of motor performance and it is shown by the scores. There were no complications so far with these procedures. Conclusions: The preliminary results indicate that DBS is highly effective, with benefit to those patients and it is according to literature.
\end{abstract}

KEY WORDS: Parkinson's disease, deep brain stimulation, subthalamic nucleous, stereotaxy.

Muitos dos sintomas da doença de Parkinson (DP) parecem ser causados pela redução na ativação do córtex motor primário, córtex pré-motor e área motora suplementar decorrentes da hiperatividade do globo pálido interno (GPI) / sustância negra pars reticulata (SNr), resultado da descarga excitatória excessiva do núcleo subtalâmico (NST). Baseados neste modelo fisiopatológico recente, muitos estudos têm demonstrado que lesão ou bloqueio da atividade do GPI ou do NST podem reverter estas anormalidades funcionais ${ }^{1-4}$. A estimulação cerebral profunda crônica (ECPC) se vale da possibilidade de obter os mesmos efeitos funcionais das ablações reduzindo as complicações permanentes, principalmente quando forem bilaterais ${ }^{5-11}$.

A história da neurocirurgia funcional para os sintomas motores da DP se confunde com a própria história de neurocirurgia. As abordagens iniciais eram feitas na área motora primária, tentando abolir principalmente o tremor e um pouco a rigidez. Passouse, a seguir, para o núcleos basais, com cirurgias abertas, na década de trinta. Com o advento da estereotaxia no meio do século passado, os alvos que nós conhecemos hoje, já eram propostos por Talairach, Guiot, Riechert e Mundinger, Leksell e tantos outros. Assim, a talamotomia ganhou forte impulso predominando sobre os demais alvos (globo pálido e subtálamo ou melhor campo de Forel ou zona incerta) e perdurou até o advento da L-Dopa. O tratamento cirúrgico a partir daí ficou restrito aos casos selecionados e a talamotomia continuou sendo o principal procedimento cirúrgico. No final da década de 80 , baseado em um novo modelo fisiopatalógico MPTP (1-metil-4-fenil-1,2,3,6-tetrahidropiridina) para a DP,

\footnotetext{
'Departamento de Neurocirurgia da Universidade Estácio de Sá, Centro de Investigação Neurológica, Instituto do Sono Estácio de Sá e Serviço de Neurocirurgia do Hospital Pró-Cardíaco, Rio de Janeiro RJ, Brasil; ${ }^{2}$ Doutorando pela Escola Paulista de Medicina/Universidade Federal de São Paulo (EPM/UNIFESP) São Paulo SP, Brasil; ${ }^{3}$ Professor Adjunto de Neurocirurgia da EPM/UNIFESP.
}

Recebido 29 Junho 2001, recebido na forma final 21 Setembro 2001. Aceito 4 Outubro 2001.

Dr. José Augusto Nasser - Avenida Ataulfo de Paiva, 1079/1001 - 22440-031 Rio de Janeiro RJ - Brasil. FAX: 2122945329.

E-mail: nasser@riodejaneiro.net 
houve o ressurgimento da palidotomia de Leksell revivido e ligeiramente modificado por Laitinen ${ }^{2,3}$. Surgiu então a possibilidade de melhorar não só o tremor e a rigidez mas também a oligocinesia e as discinesias induzidas pela L-Dopa. Assim, muitas ablações palidais foram e ainda são realizadas, com resultados bem estabelecidos na literatura. No entanto, ablação bilateral não era isenta de riscos e complicações, principalmente relacionados ao volume e à performance da fala. Além disso, a notável melhora motora não pode ser reproduzida no segundo lado operado na maioria dos pacientes. A estimulação cerebral profunda crônica (ECPC) foi inicialmente desenhada para o tratamento dos tremores, pela possibilidade de ser bilateral e passou a ser o objeto de estudo de Benabid, Lozano entre outros ${ }^{10,11}$. Estes autores, ao estimularem o GPI e o NST, mostraram que a ECPC era superior ao ganhos da ablação (palidotomia) e que também poderia haver melhora dos sintomas axiais, no que a palidotomia provou ser pouco eficiente. A vantagem da estimulação subtalâmica sobre a palidal é sutil e mais ligada à possibilidade de diminuição de medicamentos e à melhor performance motora.

Analisamos a eficácia do ECPC subtalâmica em pacientes com DP.

\section{MÉTODO}

\section{Avaliação clínica}

No período de setembro de 1999 a março de 2001, foram estudados 8 pacientes portadores de DP com mais de 5 anos de doença, com indicação de ECPC. Estes pacientes não apresentavam sinais de depressão, nem qualquer suspeita de demência. Outro critério de exclusão foi cirurgia ablativa prévia. Estes pacientes inicialmente haviam obtido boa resposta com levodopa e agonistas e agora apresentavam flutuações motoras significantes, períodos on-off frequentes, discinesias induzidas pela levodopa, hipertonia, oligocinesia marcada e tremor.

Todos os pacientes foram avaliados pelos autores no pré-operatório sob efeito de medicamento (ON) e sem efeito de medicamento por 12 horas (OFF) utilizando as escalas UPDRS, Schwab e England.

Este estudo foi aprovado pelo Conselho de Ética do Hospital Pró-Cardíaco, tendo os pacientes, após o devido esclarecimento sobre o procedimento, assinado o termo de consentimento devidamente registrado.

\section{Procedimento cirúrgico}

O primeiro tempo da cirurgia é feito sem sedação. Realizada estereotomografia, em seguida são transferidas as imagens para a central de trabalho, onde é feita a fusão destas com as imagens de ressonância magnética do cérebro do paciente feitas na véspera. Através do programa MSPS v 5.08 (Alaminos/Micromar - Diadema SP, Brasil), são feitas as fusões em tempo real. Em seguida as imagens são superpostas ao atlas neurofuncional (Shaltembrand-Wharen) para, através das coordenadas estereotáxicas específicas, localizar com precisão o núcleo subtalâmico de Luys. Alvo estereotáxico do NST: 2-5 mm abaixo da linha AC-PC, 0-4mm posterior ao ponto PMC e 9-13 $\mathrm{mm}$ lateral ao plano sagital.

Após a trepanação frontal bilateral, é posicionado um macroeletrodo (Radionics- Burllington MA, EUA) no NST e posteriormente um microeletrodo, verificando as respostas neurofisiológicas adequadas no dimídio contralateral ao procedimento e às vezes ipsilateral. Após a confirmação do melhor posicionamento da área a ser estimulada, é implantado um eletrodo quadripolar (Itrell 2 modelo 3625, Minneapolis MN, USA) e confirmado seu posicionamento final pela fluoroscopia (antero-posterior e perfil). Depois de implantado, é feita a estimulação bipolar com programador Medtronic modelo 3625, com frequências de $100-200 \mathrm{~Hz}$ e amplitude de pulso de 50-100 $\mu \mathrm{sec}$. A posição do microeletrodo é assegurada quando a estimulação induz discinesias ou quando cessa o tremor, ou quando se desenvolve contração tônica a 4,0 V. No mesmo tempo, os cabos são conectados à extensão e ao gerador de pulso subclavicular (Medtronic Itrel model 3625). Oito dias após o procedimento, é então realizada a programação inicial com programador externo Medtronic (Minneapolis MN, EUA). Todos os pacientes foram submetidos a imagem por ressonância no terceiro mês de pós-operatório confirmando o posicionamento do eletrodo (Figs 1 e 2).

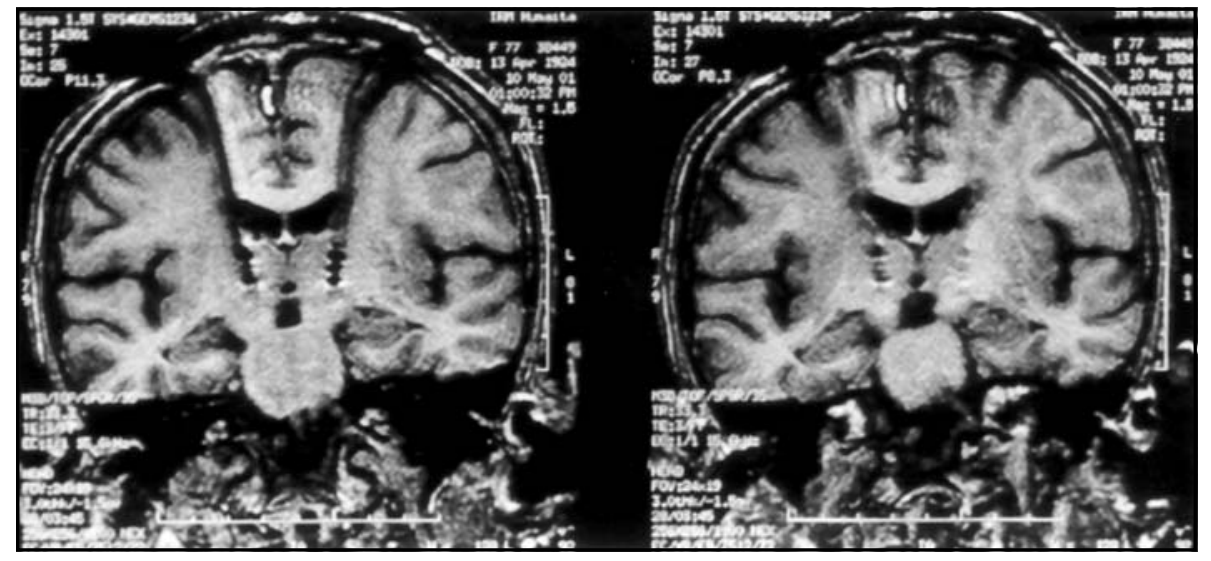

Fig 1. Ressonância magnética em T1 - Coronal mostrando o posicionamento dos eletrodos para ECPC no núcleo subcutâneo bilateral. Exame realizado no pós-operatório. 


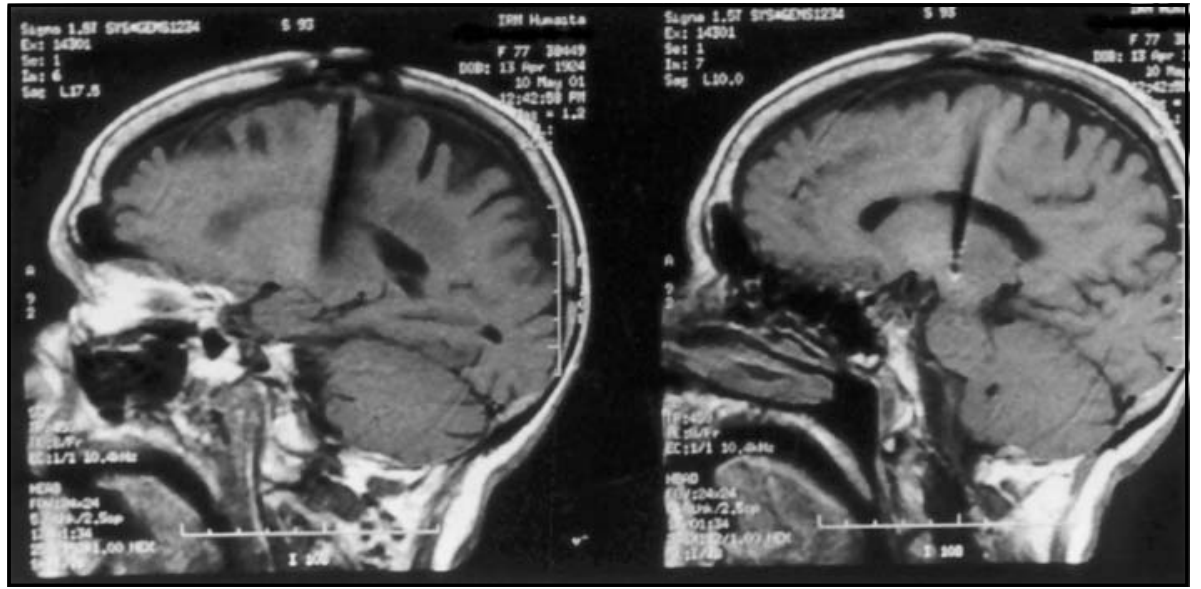

Fig 2. Ressonância magnética em T1 - sagital evidenciando o eletrodo e seus contatos no núcleo subtalâmico. Exame realizado no pós-operatório.

Tabela 1. Os oito pacientes com doença de Parkinson submetidos à estimulação subtalâmica permanente de 1999-2001.

\begin{tabular}{lcccccccc}
\hline Pacientes & 1 & 2 & 3 & 4 & 5 & 6 & 7 & 8 \\
\hline UPDRS Pré OFF & 44 & 42 & 43 & 48 & 45 & 42 & 48 & 45 \\
UPDRS Pós ON & 26 & 24 & 25 & 30 & 29 & 26 & 31 & 27 \\
UPDRS Pós OFF & 18 & 18 & 20 & 22 & 18 & 16 & 17 & 15 \\
UPDRS Pós ON & 12 & 10 & 13 & 14 & 12 & 8 & 7 & 7 \\
Schwab England Pré ON & 50 & 50 & 50 & 40 & 50 & 60 & 50 & 50 \\
Schwab England Pós ON & 70 & 80 & 70 & 70 & 70 & 80 & 80 & 80 \\
\hline
\end{tabular}

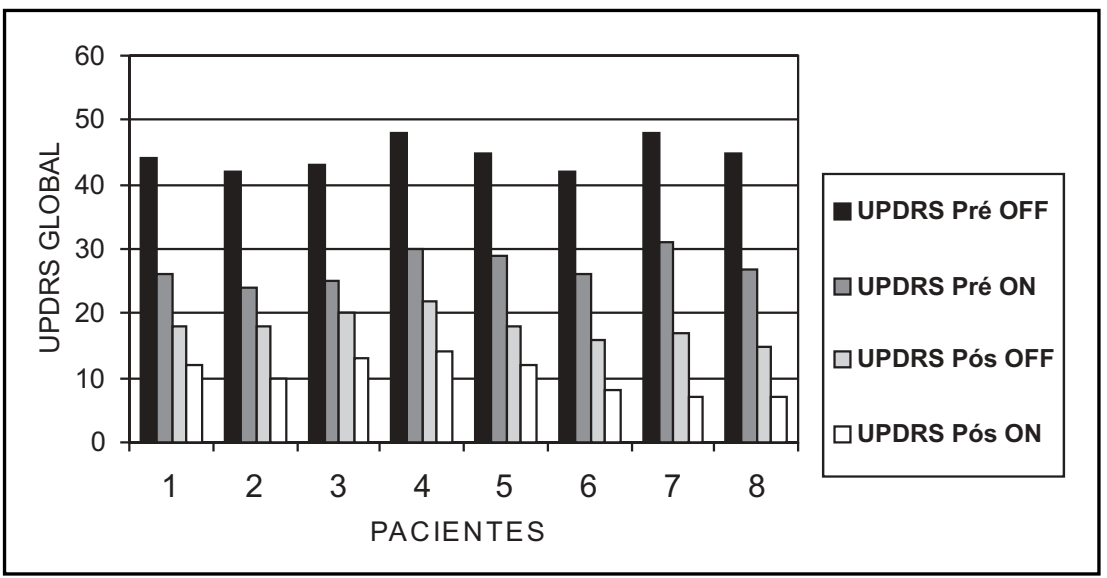

Fig 3. Análise do UPDRS com medicamento e sem medicamento no pré e no pós implante de ECPC sem medicação.

\section{RESULTADOS}

Oito pacientes foram submetidos à ECPC. A idade variou entre 60 e 74 anos (média: 67 anos). Foram 5 mulheres e 3 homens. $O$ tempo de doença variou de 7 a 12 anos (média: 9). Três pacientes tinham um lado mais comprometido, sendo 2 à direita e 1 à esquerda.

Os pacientes foram avaliados com as escalas UPDRS parte III e Schwab and England no pré-operatório e comparados com o pós-operatório sem medicação e com medicação.

Como mostram a Tabela 1 e a Figura 3, houve melhora global dos 8 pacientes. Foi observada pou- ca melhora na postura. Porém quanto ao tremor, a rigidez, a oligocinesia, a discinesia, a fala e a marcha houve melhora considerável. Nesta casuística, a melhora dos valores do UPDRS está em torno de 60\% quando avaliado sem medicação, sendo $85 \%$ para o tremor, 65\% para acinesia, 70\% para rigidez e marcha e $55 \%$ para a instabilidade. Na avaliação com medicação (ON), a melhora do UPDRS esteve em $50 \%$; houve redução significativa nas discinesias induzidas pela dopamina em torno de $80 \%$.

Apesar de ser frequente o relato de redução na medicação no período de 6 meses, apenas 2 dos nossos pacientes conseguiram redução, apenas da 
Tabela 2. Média dos parâmetros de estimulação nos 8 pacientes com ECPC.

\begin{tabular}{ll} 
Amplitude em Volts & $2.5-3.0$ \\
Largura de pulso em $\mu \mathrm{s}$ & 130 \\
Frequência em Hz & $60-90$ \\
Impedância em Ohms & acima de 2000 \\
\hline
\end{tabular}

levodopa, superior a $30 \%$ da dosagem prévia à estimulação. Em geral as flutuações motoras desapareceram ou diminuíram em intensidade.

Os parâmetros médios de estimulação estão na Tabela 2. Ao se interromper a estimulação, os tremores voltaram em todos os pacientes, assim como a bradicinesia.

Efeitos adversos apareceram em $75 \%$ dos casos, porém todos relacionados ao início da estimulação. Estes sintomas são: parestesias, disartrias e discreto aumento do tônus contralateral. Dois pacientes (25\%) se queixaram de pressão nas órbitas. Com a diminuição na amplitude (voltagem) do estimulador ou na frequência, desapareceram estes efeitos.

Não ocorreram complicações operatórias até o momento, mas não são infrequentes seus relatos na literatura, especialmente com maior número de trajetos dos microeletrodos no momento de mapear o melhor ponto dentro do núcleo subtalâmico para ser o alvo principal da microestimulação.

\section{DISCUSSÃO}

Na década de 70, Hosobushi introduziu a ECPC para dor intratável visando estimular os núcleos sensoriais do tálamo ${ }^{12}$. Em meados de 80, na França, Benabid ${ }^{9}$ iniciou ECPC para o tratamento dos pacientes com movimentos anormais, principalmente para aqueles refratários aos medicamentos e nos quais a ablação poderia ser problemática, como é o caso do tálamo, do globo pálido e subtálamo, quando há necessidade de lesão bilateral.

A ECPC se tornou realidade com o desenvolvimento de novas tecnologias aliadas à correta padronização dos parâmetros a serem adotados pelos eletrodos de estimulação. Esta técnica abria a possibilidade de estimulação bilateral, ou ainda de complementação terapêutica em pacientes operados e gravemente acometidos de sintomas não responsivos aos ensaios terapêuticos ideais ${ }^{1,10}$. A ECPC para os sintomas motores da DP evoluiu na última década, a ponto de ser possível avaliar através de estudos multicêntricos as vantagens e desvantagens de estimular o
NST sobre o GPI e também as vantagens sobre os métodos ablativos ${ }^{5,6,9}$.

Apesar de haver clara melhora nos sintomas cardinais da DP, ainda não há certeza de como a ECPC funciona ${ }^{13}$. Parece que não funciona somente como a ablação, inibindo a atividade exagerada do NST ou do GPI, mas também pelo seu efeito liberador de neurotransmissores, local e à distância, seja no estriado, na substância negra, pars reticulada e pars compacta, bem como no núcleo pedúnculo pontino $^{2,4,14,15}$.

Estimulação bilateral do NST realmente melhora as funções motoras, como mostra esta casuística em concordância com a literatura ${ }^{6}$. Segundo Limousin et al., através de estudos de imagem funcional, foi observada ativação aumentada da área motora suplementar, do córtex do cíngulo e do córtex préfrontal dorsolateral após a ECPC estar funcionando adequadamente ${ }^{13,16,17}$. É possível prever que funções cognitivas estejam sendo beneficiadas com a ECPC, como comprovam estudos recentes de neuropsicologia ${ }^{15}$.

Os benefícios da ECPC do NST em pacientes com DP avançada ocorrem mais tardiamente, sendo necessário às vezes se aguardar mais de 3 meses para poder quantificá-los. Todavia, a eficácia está bem estabelecida na literatura ${ }^{11,15}$.

\section{CONCLUSÕES}

A ECPC subtalâmica é modalidade terapêutica notável contra todos os sintomas motores da DP. Por se tratar de procedimento cirúrgico minimamente invasivo, bastante seguro e que traz poucos efeitos colaterais permanentes e pela possibilidade de implantes bilaterais simultâneos sem efeitos neuropsicológicos significantes, deve ser indicado como primeira alternativa quando o paciente é candidato à cirurgia. O fator limitante é, sem dúvida nenhuma, o econômico.

É possível reduzir em até 50\% a dosagem L-Dopa, mesmo em pacientes com tempo de doença maior que 5 anos. Deste modo, os pacientes toleram meIhor as doses menores de medicamentos e têm seus efeitos colaterais como as discinesias, menos evidentes, seja pela própria ECPC seja pela redução de doses elevadas.

Embora o mecanismo de ação da ECPC seja ainda desconhecido, advoga-se a inibição da atividade neuronal pela alta frequência.

A ECPC não influi na possibilidade de serem realizados procedimentos futuros como por exemplo, os transplantes neurais. ECPC não causa lesão ao 
redor dos contatos dos eletrodos, desde que se respeitem os parâmetros neurofisiológicos. Com a ECPC está sendo possível entender melhor os mecanismos da DP. Pela versatilidade de se poder ajustar para mais ou para menos os parâmetros de estimulação, a ECPC permite acompanhar melhor a evolução clínica do paciente com DP.

\section{REFERÊNCIAS}

1. Pollak P, Benabid AL, Limousin P, Benazzouz A. The basal ganglia and new surgical approaches for Parkinson's disease. Adv Neurol 1997;74:213-220.

2. Paré D, Curro' Dossi R, Steriade M. Neuronal basis of the parkinsonian resting tremor: a hipothesis and its implications for treatment. Neuroscience 1990;35:217-226.

3. Albin R L, Young A B, Penney JB. The functional anatomy of basal ganglia disorders. TINS 1989;12:366-375.

4. Smith Y, Kieval JZ. Anatomy of the dopamine system in the basal ganglia. TINS 2000;23:28-33.

5. Houeto JL, Damier P, Bejjani PB, et al. Subthalamic stimulation in Parkinson disease: a multidisciplinary approach. Arch Neurol 2000; 57:461-465.

6. Kumar R, Lozano AM, Kim YJ, et al. Double-blind evaluation of subthalamic nucleus deep brain stimulation in advanced Parkinson's disease. Neurology 1998;51:850-855.
7. Obeso JA, Linazadoro G, Guridi J, Ramos E. High frequency stimulation of the subthalamic nucleus and levodopa induced dyskinesias in Parkinson's disease. J Neurol Neurosurg Psychiatry 2000;68:100-126.

8. Nasser JA, Alaminos A, Ferraz A, Confort CI. Preliminary results in surgery of Parkinson's disease. Arq Neuropsiquiatr 1998,56:533-539.

9. Benabid AL, Krack P, Benazzouz A, Limousin P, Koudsie A, Pollak P. Deep brain stimulation of the subthalamic nucleus for Parkinson's disease: methodologic aspects and clinical criteria. Neurology 2000; 55(Suppl 6):40-44.

10. Pollak P, Benabid A, Limousin P, Benazzouz A. Chronic intracerebral stimulation in Parkinson's disease: the basal ganglia and new surgical approaches for Parkinson's disease. Adv Neurol 1997;74:213-220.

11. Germano IM. Chronic deep brain stimulation: indications and techniques. In Neurosurgical treatment of movement disorders. Neurosurgical topics of AANS 1998;1:159-168.

12. Hosobuchi Y.Subcortical electrical stimulation for control of intractable pain in humans: report of 122 cases (1970-1984). J Neurosurg 1986; 64:543-553.

13. Gerschlager W, Alesch F, Cunnington R, et al. Bilateral subthalamic nucleus stimulation improves frontal cortex function in Parkinson's disease: an electrophysiological study of the contingent negative variation. Brain 1999;122:2365-2373.

14. Lang AE, Lozano AM. Parkinson's disease (Part 1). N Engl J Med 1998;339:1044-1053.

15. Lang AE, Lozano AM. Parkinson's disease (Part 2). N Engl J Med 1998; 339:1130-1143.

16. Bezerra M, Martinez V, Nasser JA. Transient acute depression induced by high-frequency deep-brain stimulation. N Engl J Med 1999;341:1003-1004.

17. Zonenshayn M, Rezai AR, Mogilner AY, Beric A, Sterio D, Kelly PJ. Comparison of anatomic and neurophysiological methods for subthalamic nucleus targeting. Neurosurg 2000;47:282-294. 